\title{
Ex vivo assessment of the optical characteristics of human brain and tumour tissue
}

Shapey, Jonathan, Xie, Yijing, Nabavi, Elham, Ebner, Michael, Maneas, Efthymios, et al.

Jonathan Shapey, Yijing Xie, Elham Nabavi, Michael Ebner, Efthymios

Maneas, Shakeel R. Saeed, Neil Dorward, Neil Kitchen, Adrien E. Desjardins, Sebastien Ourselin, Zane Jaunmuktane, Sebastian Brandner, Robert

Bradford, Tom Vercauteren, "Ex vivo assessment of the optical characteristics of human brain and tumour tissue," Proc. SPIE 11251, Label-free Biomedical Imaging and Sensing (LBIS) 2020, 112510J (20 February 2020); doi: 10.1117/12.2545694

SPIE. Event: SPIE BiOS, 2020, San Francisco, California, United States 


\title{
Ex vivo assessment of the optical characteristics of human brain and tumour tissue
}

\author{
Jonathan Shapey *a,b,c,, , Yijing Xie ${ }^{c, \dagger}$, Elham Nabavic ${ }^{c, \dagger}$, Michael Ebner ${ }^{\mathrm{c}}$, Efthymios Maneas ${ }^{\mathrm{a}, \mathrm{d}}$, \\ Shakeel R Saeed ${ }^{\mathrm{b}, \mathrm{ef}}$, Neil Dorward ${ }^{\mathrm{b}}$, Neil Kitchen ${ }^{\mathrm{b}}$, Adrien E. Desjardins $\mathrm{s}^{\mathrm{a}, \mathrm{d}}$, Sebastien Ourselin ${ }^{\mathrm{c}}$, \\ Zane Jaunmuktane ${ }^{\mathrm{g}}$, Sebastian Brandner ${ }^{\mathrm{g}}$, Robert Bradford ${ }^{\mathrm{b}}$, Tom Vercauteren ${ }^{\mathrm{c}}$. \\ ${ }^{a}$ Wellcome / EPSRC Centre for Interventional and Surgical Sciences, University College London, \\ Charles Bell House, 43 - 45 Foley Street, London W1W 7TS UNITED KINGDOM; \\ ${ }^{b}$ Department of Neurosurgery, National Hospital for Neurology and Neurosurgery, Queen Square, \\ London, WC1N 3BG, UNITED KINGDOM; \\ 'School of Biomedical Engineering \& Imaging Sciences, King's College London, 1 Lambeth Palace \\ Road, London SE1 7EU, UNITED KINGDOM; \\ ${ }^{\mathrm{d}}$ Department of Medical Physics and Biomedical Engineering, University College London, Gower \\ Street, London WC1E 6BT, UNITED KINGDOM \\ 'The Ear Institute, University College London, 332 Gray's Inn Road, King's Cross, London, WC1X \\ 8EE, UNITED KINGDOM; \\ ${ }^{f}$ The Royal National Throat, Nose and Ear Hospital, London, 332 Gray's Inn Road, King's Cross, \\ London, WC1X 8EE, UNITED KINGDOM; \\ ${ }^{g}$ Department of Neuropathology, UCL Institute of Neurology, The National Hospital for Neurology \\ and Neurosurgery, Queen Square, London, WC1N 3BG, UNITED KINGDOM
}

\begin{abstract}
Emerging optical imaging techniques such as hyperspectral imaging (HSI) provide a promising non-invasive solution for intraoperative tissue characterisation with the potential to provide rich tissue-differentiation information over the entire surgical field. Neuro-oncology surgery would especially benefit from detailed real-time in vivo tissue characterisation, improving the accuracy with which boundaries of safe surgical resection are delineated and thereby improving patient outcomes. Current systems are limited by challenges with processing the HSI data because of incomplete characterisation of the optical properties of tissue across the complete visible and near-infrared wavelength spectrum. In this study, we characterised the optical properties of various freshly-excised brain tumours and normal cadaveric human brain tissue using a dual-beam integrating sphere spectrophotometer and the inverse adding-doubling technique. We adapted an integrating sphere to analyse $2 \mathrm{~mm}$-thick tissue samples measuring $4-7 \mathrm{~mm}$ in diameter and validated the experimental setup with a tissue-mimicking optical phantom. We investigated the different spectral signatures of freshly-excised tumour tissues including pituitary adenoma, meningioma and vestibular schwannoma and compared these to normal grey and white matter, pons, pituitary, dura and cranial nerve tissues across the wavelength range of $400-1800 \mathrm{~nm}$. It was found that brain and tumour tissues could be differentiated by their optical properties but the freezing process did alter the tissues' relative absorption and reduced scattering coefficients. In this work, we have demonstrated a method to characterise the optical properties of small human brain and tumour specimens that may be used as a reference dataset for developing optical imaging techniques.
\end{abstract}

Keywords: Optical properties, Tissue characterisation, Absorption, Scattering, Brain, Pituitary adenoma, Meningioma, Vestibular schwannoma

*j.shapey@ucl.ac.uk

$\dagger$ These authors contributed equally

Label-free Biomedical Imaging and Sensing (LBIS) 2020, edited by Natan T. Shaked,

Oliver Hayden, Proc. of SPIE Vol. 11251, 112510J - (C) 2020 SPIE

CCC code: $1605-7422 / 20 / \$ 21 \cdot$ doi: $10.1117 / 12.2545694$ 


\section{INTRODUCTION}

Optical imaging techniques are ideally suited for clinical use because they are non-contact, non-ionising and non-invasive. Hyperspectral imaging (HSI) is an emerging optical imaging technique with the potential to radically transform the way surgery is performed by providing real-time tissue characterisation and surgical guidance [1]. Neuro-oncology surgery would especially benefit from detailed real-time in vivo tissue characterisation, improving the accuracy with which boundaries of safe surgical resection are delineated and may improve patient outcomes.

The HELICoiD study group have demonstrated the potential of label-free HSI in guiding brain tumour surgery using customised supervised and unsupervised machine learning algorithms to classify tissue based on the target tissue's spectral signature [2][3][4][5]. However, one of the system's major limitations is its inability to deliver real-time intraoperative image analysis. To improve computational performance, an algorithmic model could benefit from known optical properties of the target tissue across the wide-wavelength spectra that HSI systems are able to capture. However, these optical properties are not yet fully known.

Reliable determination of optical properties using integrating sphere reflectance and transmittance measurements is confounded by light escaped and lost out through the edges of samples due to insufficient sample size [6]. Therefore, the size of the available tissue sample has been a major limiting factor dictating the type of tissue that could be analysed in previous studies [7], meaning that only tissue from larger tumours and large parenchymal brain regions have been characterised [8]. We addressed this limitation by adapting the integrating sphere to enable us to reliably measure the optical properties of small tissue samples measuring $4-7 \mathrm{~mm}$ in diameter.

Secondly, previous studies have only characterised the optical properties of brain tissue up to $1300 \mathrm{~nm}$ [7][8][9], whereas several HSI systems are capable of capturing spectral data up to $1800 \mathrm{~nm}$ [1]. Furthermore, previous work has focused on a limited number of glioma and meningioma tumours overlooking other tumour types such as pituitary adenomas and schwannomas. Finally, previous work has not systematically examined whether or not there is a difference between the optical properties of fresh and frozen tissue samples.

In this study, we describe the adaptation and validation of a dual-beam integrating sphere system to perform spectroscopic measurements of small biological human tissue samples. We investigated: 1) the optical absorption and reduced scattering properties of various ex vivo human brain tumour tissues including meningioma, pituitary adenoma and vestibular schwannoma and healthy brain tissue including white matter, grey matter, dura, pons, anterior pituitary gland and cranial nerves; and 2) whether the process of freezing tissue prior to analysis altered its relative absorption and reduced scattering coefficients.

\section{METHODS AND MATERIALS}

\subsection{Integrating sphere measurements}

A dual-beam spectrophotometer (Lambda 750, Perkin Elmer, USA) with a $100 \mathrm{~mm}$ integrating sphere was adapted to measure the tissues' reflectance and transmittance spectra in the wavelength ranging from $400 \mathrm{~nm}$ to $1800 \mathrm{~nm}$. The standard sample reflectance port dimensions are $17 \mathrm{~mm} \times 22 \mathrm{~mm}$ and the transmittance port dimensions are $11 \mathrm{~mm} \times 24$ $\mathrm{mm}$. However, based on the size of pathology specimens typically obtained at our institution, we anticipated that a sample port size of $3 \mathrm{~mm}$ to $5 \mathrm{~mm}$ in diameter would be required. To meet these specifications, we adapted the integrating sphere using an adjustable light slit and a series of uncoated plano-concave lenses to collimate and focus the light appropriately thereby ensuring that the eventual beam size was smaller than the anticipated sample size. Bespoke adapters with smallsized sample ports to hold the tissue slides were 3D printed and tissue slides were manually manufactured using $2 \mathrm{~mm}$ thick acrylic. The experimental setup was validated with a tissue-mimicking optical phantom previously characterised with the same machine using standard sample reflectance and transmittance ports and our results were in agreement with previously characterised data [10].

\subsection{Subjects}

This study was approved by the NHS Health Research Authority (18/LO/1004). Patient tumour samples were obtained from the Brain Tumour Resource at the Department of Neuropathology, Queen Square. All samples came from adult patients who underwent brain tumour surgery at the National Hospital for Neurology and Neurosurgery (NHNN) who 
consented to their tissue being stored and used in future research studies. Tissue samples were obtained from 8 different patients undergoing brain tumour surgery including 3 meningioma, 3 pituitary adenoma and 2 vestibular schwannoma. All tumour specimens were obtained fresh and processed within 30 minutes following resection.

Cadaveric healthy brain tissue was obtained from patients undergoing a post mortem examination at the Department of Neuropathology, NHNN, who had a valid consent form authorising the retention of tissue for ethically approved research purposes. Tissue samples from 9 distinct anatomical regions was obtained from a single healthy cadaver including frontal grey matter, frontal white matter, anterior pituitary, pituitary stalk, pons, cranial nerve II, cranial nerve III, cranial nerve V and dura.

In total, 126 paired reflectance and transmittance measurements were performed.

\subsection{Sample preparation and analysis}

All specimens were processed according to the study protocol. For each tumour specimen, 4 samples were obtained; 2 for fresh analysis and 2 for analysis after freezing. The maximum sized tissue was selected, and the specimen was placed in the chamber of a suitably sized slide ensuring that the tissue completely covered the central $3 \mathrm{~mm}$ or $5 \mathrm{~mm}$ hatched area. All specimens were $2 \mathrm{~mm}$ thick. Index matching gel (Thorlabs, Cat no: G608N3) was used to secure the tissue in place by filling the empty region within the chamber at either end of the specimen. Frozen specimens were immediately snap frozen using Frosbite rapid coolant spray (Leica Microsystems (UK) Ltd). A small amount of Pertex mounting medium was applied to the sides of the specimen chamber and a coverslip was placed on top of the specimen to seal the specimen within the central chamber. Frozen specimens were stored at $-80{ }^{\circ} \mathrm{C}$ and analysed at least 7 days later, once thawed at room temperature for at least 30 minutes. Three reflectance and transmittance measurements were obtained for each sample.

\subsection{Data processing technique}

An inverse adding-doubling (IAD) technique developed by Prahl et al. [11] was used to determine the optical absorption and reduced scattering coefficients from the mean reflectance and transmittance spectra for each sample. As estimated in previous studies, the refractive indices of the sample tissue and glass slides were estimated to be 1.40 and 1.50 respectively [7] and since collimated transmittance spectra were not obtained in these measurements, a fixed anisotropy coefficient $(\mathrm{g}=0.85)$ was assumed for every wavelength and sample [7][9]. Absorption and reduced scattering coefficients were computed for each specimen and used to calculate mean and standard error of the mean (SEM) values for each tissue type. Data that did not converge using our specified constraints were excluded when calculating the mean values.

\section{RESULTS}

\subsection{Optical spectra of ex vivo brain tumour tissue}

Figure 1 illustrates the mean absorption $\left(\mu_{\mathrm{a}}\right)$ and reduced scattering $\left(\mu^{\prime}\right.$ s) coefficient spectra of all tumour samples. All tumour types shared qualitatively similar dependencies of the optical properties on the wavelength but in a small number of samples IAD analysis did not converge in the wavelength range of 800 to $1300 \mathrm{~nm}$. The absorption spectra of all samples had a series of peaks below $600 \mathrm{~nm}$ and above $900 \mathrm{~nm}$ typically associated with the absorption bands of haemoglobin and water respectively [12], with low absorption values within the intervening $600 \mathrm{~nm}$ to $900 \mathrm{~nm}$ wavelength range. The absorption spectra of all three tumour types also demonstrated peak absorption bands around $430 \mathrm{~nm}$ and 550 $\mathrm{nm}$ characteristic of oxy- and deoxy-haemoglobin, respectively [13].

The reduced scattering coefficient spectra of all tumours generally decreased with increasing wavelength, consistent with Rayleigh limit of Mie scattering. All tumour spectra contained two modest scattering peaks at $430 \mathrm{~nm}$ and $550 \mathrm{~nm}$ consistent with the corresponding haemoglobin absorption spectra but, most strikingly, all three tumour types also exhibited a very prominent peak in $\mu^{\prime}$ s at $1450 \mathrm{~nm}$, corresponding to a similarly pronounced water-related absorption peak at the same wavelength. In between these peaks in $\mu^{\prime}$ s, the three different tumour types could be differentiated by the strength of their reduced scattering spectra. In particular, meningioma tissue displayed consistently higher $\mu_{\text {s }}^{\prime}$ values compared to schwannoma tissue. However, the reduced scattering spectra of fresh pituitary tumour tissue was highly variable, making it difficult to draw firm conclusions relating to the $\mu$ 's of pituitary tumours. 
Within the wavelength range of $700-1100 \mathrm{~nm}$, the differentiating water peaks seen in the absorption spectra of fresh tumour tissue appeared less pronounced in the frozen samples, although the $\mu_{\mathrm{a}}$ spectra above $1200 \mathrm{~nm}$ appeared largely similar. These results suggest that the freezing process does affect the absorption and reduced scattering spectra of all tissues, particularly within the $700-1100 \mathrm{~nm}$ wavelength range.
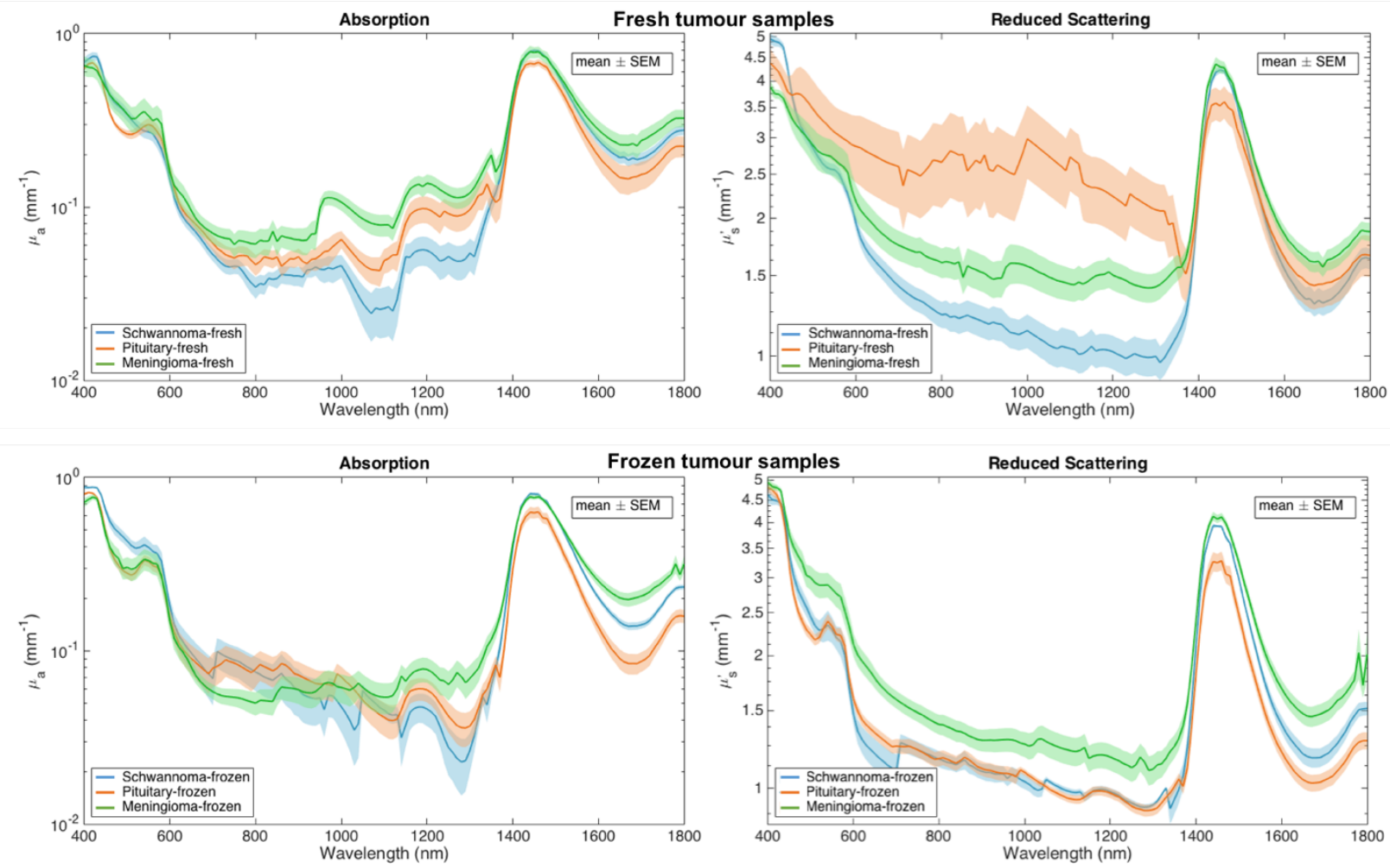

Figure 1. Optical absorption $\left(\mu_{\mathrm{a}}\right)$ and reduced scattering $\left(\mu_{\mathrm{s}}^{\prime}\right)$ coefficient spectra of all tumour samples. Blue: Schwannoma, Orange: Pituitary, Green: Meningioma. Line: mean value. Shaded area: Standard Error of the Mean (SEM). Mean values plotted with SEM values for all samples. Top row: Fresh samples. Bottom row: Frozen samples.

\subsection{Optical spectra of healthy cadaveric brain tissue}

Figure 2 illustrates the absorption and reduced scattering spectra of all thawed cadaveric healthy brain tissue samples. As observed in tumour tissue, healthy brain tissue also displayed similar absorption peaks related to the presence of haemoglobin and water in addition to the expected low absorption values within the wavelength range of $600 \mathrm{~nm}$ to 900 $\mathrm{nm}$. The reduced scattering spectra of healthy brain tissue also demonstrated typical decreasing values with increasing wavelength. Similar to the tumour samples, healthy brain tissue may be differentiated by their respective reduced scattering spectra. The relative levels of reduced scattering were highest for white matter at all investigated wavelengths. However, the peak at $1450 \mathrm{~nm}$ seen in other brain tissue types was far less marked in white matter. Pons tissue demonstrated a spectral curve between that of grey and white matter and the reduced scattering spectra of cranial nerves was lower than that of parenchymal brain tissue. 

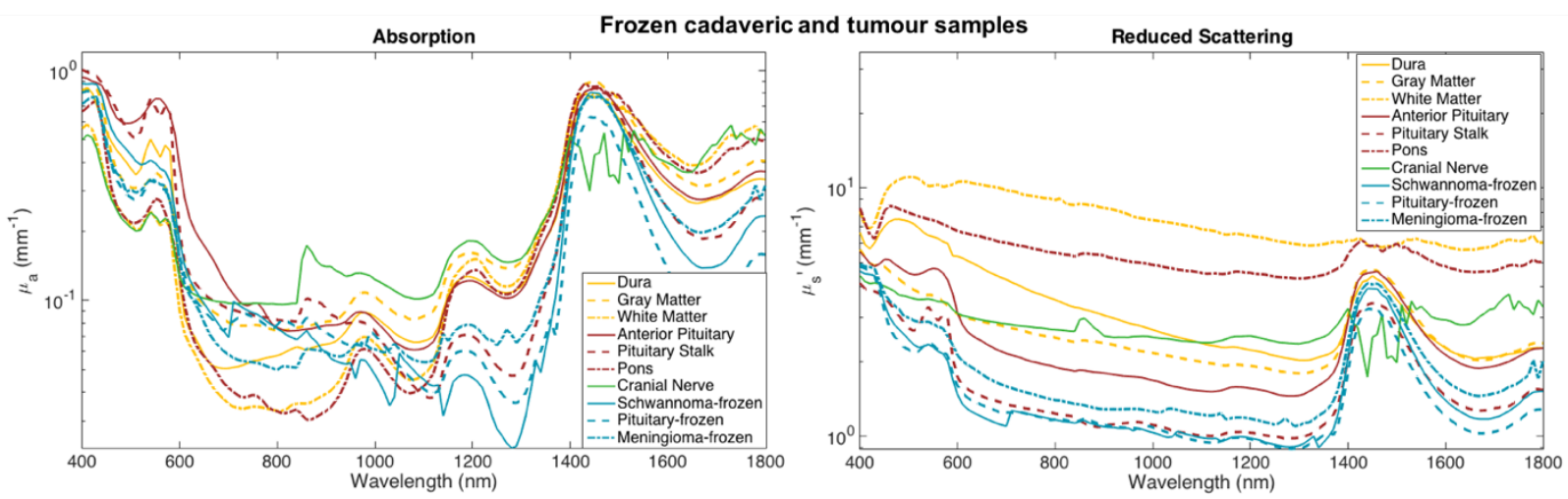

Figure 2. Mean values of absorption $\left(\mu_{\mathrm{a}}\right)$ and reduced scattering $\left(\mu_{\mathrm{s}}^{\prime}\right)$ coefficient spectra of all frozen cadaveric healthy brain tissue and frozen tumour samples.

\section{DISCUSSION AND CONCLUSION}

In this study, we investigated the ex vivo optical properties of three types of human brain tumour and various different types of healthy brain tissue across a wide wavelength spectrum of $400-1800 \mathrm{~nm}$. Previous studies have examined the optical properties of parenchymal brain tissue [9][7][8], glioma [9][7][14] and meningioma [7] up to $1300 \mathrm{~nm}$ but to the best of our knowledge this is the first study to investigate their optical properties beyond $1300 \mathrm{~nm}$. We investigated the optical properties of meningioma, pituitary adenoma and vestibular schwannoma and correlated these with the absorption and reduced scattering spectra of related anatomical tissues including frontal grey and white matter, pituitary tissue, pons, cranial nerves and dura. No previous studies have examined the optical properties of pituitary adenoma or vestibular schwannoma tissue and this is the first study to examine the optical spectra of healthy pituitary tissue, cranial nerves and dura. Additionally, we systematically analysed what effect the freezing process might have on the optical properties of tumour tissue samples.

We observed that the spectral curves of the three types of examined brain tumour had a similar morphology to those of normal cadaveric human brain tissue. Similar to previous studies, the absorption spectra of all samples were dominated by the absorption bands of haemoglobin below $600 \mathrm{~nm}$ and water above $900 \mathrm{~nm}$, with characteristic low absorption values within the 600 to $900 \mathrm{~nm}$ wavelength range [7][9]. However, our results demonstrated a further striking peak in $\mu_{\mathrm{a}}$ at $1450 \mathrm{~nm}$, not previously reported, but again consistent with the absorption spectra of water [15]. The reduced scattering coefficient spectra of all tissues generally decreased with increasing wavelength, while the values of reduced scattering were very different across all tissue types. These findings are in line with the expected combination of Rayleigh and Mie scattering but as noted in previous studies [7][9], inter-parameter 'crosstalk' between the tissue's relative absorption and reduced scattering coefficients did occur in wavelengths with high absorption values causing local maxima in the scattering spectra. Given that the highest absorption peak occurred at $1450 \mathrm{~nm}$, it is therefore not surprising that the highest degree of crosstalk also occurred at this wavelength. This phenomenon was most pronounced in cortical grey matter and pons tissue but was also seen in healthy dura and pituitary tissue and to a lesser degree in the tumour samples. This may be explained by the fact that those tissues exhibiting this phenomenon are predominantly composed of cell bodies whereas white matter consists predominantly of myelinated axons.

We also investigated what effect the freezing process might have on the tissues' optical properties using tumour samples. Roggan et al. and Mesradi et al. previously observed that snap-freezing porcine and rat tissue produced significant changes in the tissues' absorption and reduced scattering coefficients [16][17]. However, in their study examining the optical properties of human brain tissue, Gebhart et al. performed a comparison study of four tissues samples and concluded that "snap freezing, tissue storage and thawing in saline had minimal effect on the measurement absorption and reduced scattering coefficients" [7]. We performed a systematic experiment comparing the optical properties of two fresh and two frozen tumour samples taken from each specimen. As illustrated in Figure 1, our results suggest that the freezing process did affect the sample's absorption coefficient, particularly within the wavelength range of $700-1100 \mathrm{~nm}$ but its effect was less significant above $1200 \mathrm{~nm}$.

In conclusion, we measured the optical properties of various ex vivo human brain tumour tissues including meningioma, pituitary adenoma and vestibular schwannoma and several distinct healthy brain tissues across the wavelength range of 
$400-1800 \mathrm{~nm}$. It was found that brain and tumour tissues could be differentiated by their optical properties but the freezing process did alter the tissues' relative absorption and reduced scattering coefficients.

\section{ACKNOWLEDGEMENTS}

This work was supported by Wellcome Trust [203145Z/16/Z; 203148/Z/16/Z; WT106882], EPSRC [NS/A000050/1; NS/A000049/1] and National Brain Appeal [NBA/T\&I/N-ONC] funding. TV is supported by a Medtronic / Royal Academy of Engineering Research Chair [RCSRF1819\7\34].

\section{REFERENCES}

[1] J. Shapey et al., "Intraoperative multispectral and hyperspectral label-free imaging: a systematic review of in vivo clinical studies," J. Biophotonics, p. e201800455, Mar. 2019.

[2] H. Fabelo et al., "HELICoiD project: A new use of hyperspectral imaging for brain cancer detection in real-time during neurosurgical operations," in Proceedings of SPIE - The International Society for Optical Engineering, 2016, vol. 9860.

[3] D. Ravi, H. Fabelo, G. M. Callico, and G. G.-Z. Yang, "Manifold Embedding and Semantic Segmentation for Intraoperative Guidance with Hyperspectral Brain Imaging," IEEE Trans. Med. Imaging, vol. 36, no. 9, pp. 1-1, Sep. 2017.

[4] H. Fabelo et al., "Spatio-spectral classification of hyperspectral images for brain cancer detection during surgical operations.," PLoS One, vol. 13, no. 3, p. e0193721, 2018.

[5] H. Fabelo et al., "An Intraoperative Visualization System Using Hyperspectral Imaging to Aid in Brain Tumor Delineation.," Sensors (Basel)., vol. 18, no. 2, Feb. 2018.

[6] J. W. Pickering, S. A. Prahl, N. van Wieringen, J. F. Beek, H. J. C. M. Sterenborg, and M. J. C. van Gemert, "Double-integrating-sphere system for measuring the optical properties of tissue," Appl. Opt., vol. 32, no. 4, p. 399, Feb. 1993.

[7] S. C. Gebhart, W. C. Lin, and A. Mahadevan-Jansen, "In vitro determination of normal and neoplastic human brain tissue optical properties using inverse adding-doubling.," Phys. Med. Biol., vol. 51, no. 8, pp. 2011-27, Apr. 2006.

[8] S. Madsen and B. Wilson, Optical Properties of Brain Tissue, vol. 3. New York: Springer, 2013.

[9] A. N. Yaroslavsky, P. C. Schulze, I. V Yaroslavsky, R. Schober, F. Ulrich, and H.-J. Schwarzmaier, "Optical properties of selected native and coagulated human brain tissues in vitro in the visible and near infrared spectral range," Phys. Med. Biol., vol. 47, no. 12, p. 305, Jun. 2002.

[10] E. Maneas et al., "Gel wax-based tissue-mimicking phantoms for multispectral photoacoustic imaging," Biomed. Opt. Express, vol. 9, no. 3, pp. 1151-1163, Mar. 2018.

[11] S. A. Prahl, M. J. van Gemert, and A. J. Welch, "Determining the optical properties of turbid mediaby using the adding-doubling method," Appl Opt, vol. 32, no. 4, pp. 559-568, 1993.

[12] S. L. Jacques, "Generic tissue optical properties," 2015. .

[13] S. Prahl, "Optical absorption of hemoglobin," 1999. .

[14] H. J. C. M. Sterenborg, M. J. C. Van Gemert, W. Kamphorst, J. G. Wolbers, and W. Hogervorst, "The spectral dependence of the optical properties of human brain," Lasers Med. Sci., vol. 4, no. 4, pp. 221-227, Dec. 1989.

[15] V. Nguyen, Y. Paulus, V. P. Nguyen, and Y. M. Paulus, "Photoacoustic Ophthalmoscopy: Principle, Application, and Future Directions," J. Imaging, vol. 4, no. 12, p. 149, Dec. 2018.

[16] A. Roggan, D. Schädel, U. Netz, J. P. Ritz, C. T. Germer, and G. Müller, "Effect of preparation technique on the optical parameters of biological tissue," Appl. Phys. B Lasers Opt., vol. 69, no. 5, pp. 445-453, Nov. 1999.

[17] M. Mesradi et al., "Experimental and analytical comparative study of optical coefficient of fresh and frozen rat tissues," J. Biomed. Opt., vol. 18, no. 11, p. 117010, Nov. 2013. 